\title{
Understanding toxicity in multiplayer online games: The roles of national culture and demographic variables
}

\author{
Bastian Kordyaka \\ University of Luebeck \\ bastian.kordyaka@uni-luebeck.de
}

\author{
Jeanine Krath \\ University of Koblenz-Landau \\ jkrath@uni-koblenz.de
}

\author{
Solip Park \\ Aalto University \\ solip.park@aalto.fi
}

\author{
Henrik Wesseloh \\ University of Göttingen \\ henrik.wesseloh@uni-goettingen.de
}

\begin{abstract}
Toxic behavior (TB) is a negative response to in-game frustration in multiplayer online games (MOG) that can ruin the playing experience, causing financial damage to MOG operators. Understanding the drivers of TB is an important step to curb the behavior. In this work, we consult the model of national culture (MNC) as well as demographic variables (e.g., education, gender, and age) as antecedent variables of TB using an exploratory design. We surveyed players of League of Legends and Dota 2 with two samples, based on the MNC, from North America $(n=155)$ and India $(n=119)$. We observed significant cultural differences in $T B$, with higher levels of self-reported toxicity in the Indian sample. In both samples, consistent with previous findings, age was negatively associated with TB. However surprisingly, there was a statistically significant difference among the two groups in terms of the relationship between education and TB.
\end{abstract}

\section{Introduction}

Multiplayer online games (MOGs) such as Fortnite, Valorant, Starcraft, Dota 2 and League of Legends (LoL) are played by millions of people around the world every day. The video game industry is experiencing steady growth, and according to Newzoo and Statista, its global market value was approximately $\$ 159$ billion in 2020 [1]. Gaming can be an enjoyable, harmonious pastime, and the social functions of these games bring people together for meaningful interactions [2, 3]. However, negative behaviors such as cyberbullying or toxic behavior (TB) pose a threat to positive online social outcomes $[4,5]$. Since TB can ruin the gaming experience for others, it can even lead to loss of revenue for some game developers whose game suffers greatly, as it can deter new players from playing the game $[4,6]$.

\author{
Samuli Laato \\ Tampere University \& University of Turku \\ samuli.laato@tuni.fi
}

In addition, developers can be seen as having an ethical responsibility to protect underage players in particular from excessive profanity and abuse in their game [7]. Consequently, it is important to understand the drivers of TB in order to develop effective countermeasures.

$\mathrm{TB}$ in online games differs from cyberbullying in that it is: (1) impulsive and of short duration; and (2) a reaction to in-game frustration $[4,5]$. In the context of MOBA games such as Dota 2, TB can manifest through gameplay (e.g., intentionally stealing resources from teammates) and through team communication in the form of verbal and written abuse [4]. Previous research already showed that $\mathrm{TB}$ is influenced by a variety of factors, highlighting the role of game design and in-game events [8,9] as well as personality and attitudinal factors $[10,11]$. The impact of offline culture and demographic variables has been less studied in the extant literature. However, it is essential to consider these factors because they provide the opportunity to build efficient target group appropriate countermeasures on a level of game design. For example, since quantitative video game research often relies on samples from specific countries all around the world, it is necessary to investigate what kind of influence the culture specific to certain countries exerts on the occurrence of TB.

To address this research gap, we approach TB in MOGs via two quantitative samples $(n=155$ and $\mathrm{n}=119$ ) originating from two countries that differ in terms of their cultural dimensions [12]. On a level of theory, we draw on the model of national culture (MNC) [13]. According to the MNC, cultures can be distinguished based on six different dimensions (power distance, individualism/collectivism, masculinity, uncertainty avoidance, long term orientation and indulgence) $[13,12]$. In our case, we compare a culture low in collectivism and power distance and high in indulgence (i.e., NA) with a culture high in collectivism and power distance and low in indulgence 
(i.e., India) in view of TB and the exploratory potential of demographic variables. As cases of application we refer to two of the most successful MOGs League of Legends and Dota 2. With this design, we attempt to provide answers to the following two research questions:

Research Question 1: How does the frequency of toxicity differ between different cultural settings?

Research Question 2: What influence do demographic variables have on the occurrence of toxicity in different cultural settings?

To answer our research questions the remainder of this study is organized as follows. First, in the Background section, we review previous work on TB in MOGs, discuss the effects and antecedents of TB, and our theoretical framework, the MNC [13]. Next, we describe the empirical research methodology and data collection. Thereafter, we present the results of our analysis, followed by a discussion of our main findings, their implications and limitations, future work, and conclusions.

We observed quantifiable differences in self-reported TB as well as in preference for game type between national cultures. In addition, age had a negative influence on TB in both cultures, whereas in contrast, education had a positive effect on TB only in the NA group. While previous work predominantly focused on situational conditions or motivations as antecedents of TB, our work shows that dispositional determinants such as culture, age and education are very important to explain variances in TB. Consequently, game designers should consider region-specific solutions and strategies as well as educational opportunities and encouragement of personal growth in their games to mitigate TB in global MOGs.

\section{Background}

\subsection{Toxic behavior in multiplayer online games}

TB, or toxicity, is a common concomitant in MOGs [14, 9]. It encompasses of verbal or non-verbal harassment or intentional triggering of teammates and thereby can constitute of a variety of different negative behaviors, such as flaming and griefing $[5,9,15,8,11$, 16]. In contrast to cyberbullying, which is an intentional and aggressive form of personal harassment in the online environment, TB is characterized by being temporal and primarily an attempt to cope with frustration as a specific form of external attribution [4, 17] (see Table 1).

However, the measurement of $\mathrm{TB}$ is still in the early stages of development and especially in cases of non-verbal TB, there is a great chance of false positives and false negatives in identifying TB. Sometimes players may unintentionally behave in ways that trigger fellow players. This phenomenon is exacerbated by the fact that MOGs host players from different cultures, age groups and educational backgrounds. Because of the difficulty in accurately identifying TB by observing players' actions alone, the majority of validated instruments developed to measure TB rely on asking players about their own perceptions in case of a situation in which they get mad [4], e.g. if they insult other players (verbal harassment, flaming), take away resources belonging to other players (non-verbal harassment, griefing) or intentionally interrupt other players while they are writing (intentional triggering of teammates) [4]. Nonetheless, a recent study triangulated behavioral and self-reported TB showed substantial reliability between both sources of data [4].

Previous research on TB in MOGs has shown several harmful effects associated with TB in the virtual environment. The presence of a single toxic player can critically affect team cohesion [20] and overall team performance $[14,15,16,20]$ by causing stress and conflict among team members. Although TB is mostly a temporary phenomenon, it has been shown to be contagious among gamers $[15,8]$. Research suggests that continuous exposure to $\mathrm{TB}$ in certain online environments can cause psychological and emotional problems such as anxiety and low self-esteem [4, 5, 21]. Consequently, game developers attempt to curb TB [5, $7,22]$, however this is not straightforward. Applied strategies and approaches include introducing reporting features $[16,22]$, penalties for misbehaving players $[17$, $22]$, providing muting and blocking features to enable silencing players [22] and in-game nudges toward collaborative behavior [22]. Nevertheless, players still consider TB in MOGs to be a severe problem that may even have worsened since the onset of the COVID-19 pandemic [23]. Hence, it is important to advance scientific understanding of the causes of TB in order to develop effective countermeasures and mitigate the negative outcomes.

\subsection{Antecedent variables of toxic behavior}

Previous research on the factors influencing TB in MOGs can be broadly divided into studies addressing situational conditions that promote the occurrence of TB and work addressing personal dispositions that facilitate TB. Regarding the former, highly competitive ranked games seem to significantly reinforce TB $[6,9,11,16]$. In addition, high skill disparities within teams and 
Table 1. Classification of negative behavior (adapted from [4])

\begin{tabular}{llc}
\hline Construct & Definition & Duration \\
\hline Bullying & ...an intentional behavior harassing, offending, socially & Repeating \\
& $\begin{array}{l}\text { excluding or negatively affecting someone [18] } \\
\text {... an aggressive intentional behavior carried out by a group or }\end{array}$ & Repeating \\
Cyberbullying & an individual, using electronic forms of contact [19] & Temporary \\
Toxic behavior (includes flaming, & ..a behavior generating anger and frustration in players, & \\
verbal abuse \& griefing) & $\begin{array}{l}\text { harming communication, and contributing to spreading a bad } \\
\text { mood [15] }\end{array}$ & \\
\hline
\end{tabular}

substantial weekly playing time among team members favor the presence of TB [8, 11]. These situational factors potentially catalyze tilting, a cyclical process of negative behavior and emotions as a result of poor game performance that drives players into TB $[9,16]$. It has been shown that only $1 \%$ of players in the game League of Legends are consistently toxic, while the vast majority of reported TB is attributable to such situational factors [24]. In addition, TB is often fuelled by the natural anonymity on the internet and the impossibility to connect game and real-world identities (despite playing with known acquaintances, which only accounts for a small fraction of TB), a phenomenon discussed as the online disinhibition effect [25]. Accordingly, previous research showed the substantial influence of negative disinhibition (the dark side of disinhibition) as a predictor of the occurrence of TB [5].

In addition, previous work suggests that predispositions such as attitudes [5], own victimization experiences [5], behavioral control [5], and motivations $[11,10]$ influence TB in MOGs, along with one study finding that men are more likely to engage in TB than women, and that younger age seems to promote TB [11]. Still, the dispositional determinants of TB and the influence of the player's real-world environment require further investigation. Following up on the interesting finding by Kwak et al. [17] showing that TB differs significantly by server region, we aim to contribute to the scientific understanding of the determinants of TB by investigating the influence of players' cultural background on the occurrence of TB.

\subsection{Culture and related considerations in video games}

Games are creative cultural production goods that are intricately interplaying with economic, governmental, and infrastructural extensions of digital platforms and ecosystems [26, 27]. Previous studies have acknowledged digital games as a new rising cultural media phenomenon [28], a channel for knowledge acquisition [29, 30], the virtual stage of cultural identity and commodity [31, 26, 32], and social representation and interactions [33]. As such, the necessity of studying the link between the culture and games has emerged in academia [34], with growing interest in the process of construction of those video game cultures [35].

National and generational cultures are very visible in the video game industry. Cultural factors related to, for example, players' genre and gaming platform preferences, their spending behaviors, and player communities and their behavior need to be considered in global game marketing and sales [31, 36]. Meanwhile many game studies have emphasized an in-depth analysis of one specific country, game, or region. However, it is important to acknowledge that today digital game play is a transnational cultural phenomenon [37] and is a fusion between national and global cultures [38] that often accommodates cross-cultural encounters [39]. For example, a study conducted on the North American community of competitive Halo 3 players showed a degree of homogenization across localized sites while playing the games globally, and asserts the limits of studying gaming primarily as a local phenomenon [40]. Another related work, presented evidence of cultural translocalism and hybridization within a personal, institutional, and cultural dimensions of digital game consumption and development in Eastern Europe and the Middle East [39].

Despite the wide dissemination in other research encounters, the variable culture has not been used in the context of TB beyond preliminary work [17], which is surprising because target group specific game design can substantially increase the efficiency of existing countermeasures. To capture culture, we refer to a well-established $\mathrm{MNC}[13,12]$, which identifies the following six dimensions that differ between human cultures: (1) power distance (e.g., the expectation that power is distributed unequally); (2) individualism/collectivism (e.g., the expectation that individuals take care of only themselves and their immediate families/ the expectation individuals can 


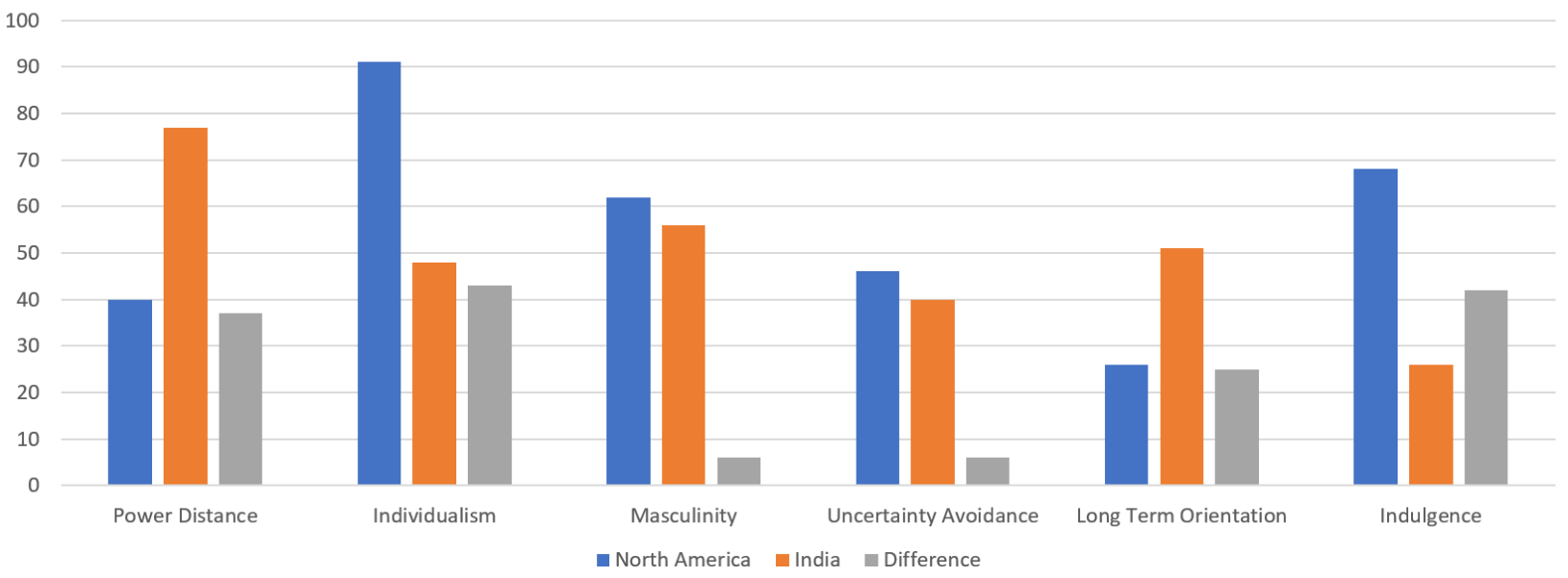

Figure 1. The dimensions of the model of national culture [12] in relation to NA and India

expect their relatives or members of a particular ingroup to look after them); (3) masculinity (e.g., represents the preference for achievement, heroism, assertiveness, and material rewards); (4) uncertainty avoidance (e.g., represents the degree to which the members of a society feel uncomfortable with uncertainty and ambiguity); (5) long term orientation (e.g., represents the encouragement for thrifts and efforts in modern education as a way to prepare for the future); and (6) indulgence (e.g., represents the preference for a relatively free gratification of basic and natural human drives related to enjoying life and having fun).

For the purposes of our study, we compare the cultures of North America (NA) and India. First, we can see notable distinctions in the gaming platform preferences of the regions. North America, which is one of the dominant exporters of video games in the global gaming market, has solidified its position with a strong traditional preference for console and PC-based games to date [41]. In contrast, many emerging countries in Asia-Pacific regions, including India, have witnessed the emergence of a highly mobile-centric gamer culture in recent years. For example, $89 \%$ of gaming revenue generated in India derives from mobile games, with $40 \%$ of its gamers spending more than six hours of active gameplay per week [42].

Second, using the MNC [12], we can justify the differentiation of India and NA based on substantial differences in terms of the cultural dimensions of power distance, individualism, and indulgence (see Figure 1) ${ }^{1}$. Based on this evidence, players in India should exhibit

\footnotetext{
${ }^{1}$ The values for the Table were retrieved from https://www.hofstede-insights.com/product/compare-countries/ on 4th of June, 2021
}

higher power distance and lower levels of individualism and indulgence.

\section{Materials and methods}

\subsection{Research design}

To get relevant insights regarding our two research questions, we used a cross-sectional survey approach and collected self-reported data from players using an online questionnaire. Subsequently, we analyzed the data with covariance-based statistics (i.e., t-tests and regression analysis) using the software SPSS 27 to better understand the influence of the cultural dimensions and demographics in the context of TB in MOGs.

\subsection{Constructs and other variables}

Toxic behavior. To measure TB, we used an empirically validated scale consisting of five items $(M=3.87, S D=1.76$; [4]). The scale used a seven-point Likert scale ranging from 1 ("strongly disagree") to 7 ("strongly agree"). We decided to use the average sum scores of the factors of the relevant items because they could be averaged to reflect the scale, are easy to interpret, and preserve the initial variation in the data [43]. In our data the scale showed good internal consistencies (Cronbach's Alpha 0.93).

Cultural dimensions. To distinguish between the two cultures of NA and India, we drew on the theoretical perspective of MNC [12] and selected the three cultural dimensions in which the two countries differed most (see Figure 1). These were (1) power distance; (2) individualism vs collectivism; and (3) indulgence. 
Table 2. Demographic information based on culture

\begin{tabular}{lcccc}
\hline Sample 1: NA (n=155) & $\min$ & $\max$ & M & SD \\
\hline Age & 20 & 64 & 31.0 & 8.10 \\
Playtime (hours/week) & 1 & 35 & 6.74 & 5.63 \\
Playing xp (years) & 1 & 12 & 4.80 & 7.64 \\
\hline Gender & Sum & & Total & \\
\hline Male & 100 & & & \\
Female & 55 & & 155 & \\
\hline Education & Sum & & Total & \\
\hline Some high school & 1 & & & \\
High school graduate & 7 & & & \\
Some college credit & 18 & & & \\
Technical training & 3 & & & \\
Associate degree & 21 & & & \\
Bachelor's degree & 78 & & & \\
Master's degree & 25 & & & \\
Doctorate degree & 2 & & 155 & \\
\hline Sample 2: India (n=119) & & & & \\
\hline Age & 19 & 42 & 27.0 & 4.11 \\
Playtime (hours/week) & 1 & 40 & 9.51 & 9.20 \\
Playing xp (years) & 1 & 12 & 2.91 & 1.94 \\
\hline Gender & Sum & & Total & \\
\hline Male & 85 & & & \\
Female & 34 & & 119 & \\
\hline Education & Sum & Total & \\
\hline High school graduate & 1 & & & \\
Some college credit & 2 & & & \\
Bachelor's degree & 93 & & 119 & \\
Master's degree & 23 & & & \\
\hline & & & & \\
\hline
\end{tabular}

Additionally, we collected various demographic (e.g., age, gender, education) and control variables (e.g., playtime, playing experience, game type) as potential antecedent variables of TB.

\subsection{Data collection and participants}

We conducted a survey collecting data from 305 multiplayer video game players using the crowdsourcing marketplace Mechanical Turk (MTurk). All participants received \$1.89 as a reward for participating in the study. To ensure that participants followed the requirement of playing one of the two relevant games in our study (i.e., LoL or Dota 2), we asked them to indicate their three favorite game characters and excluded participants with spurious answers ( 15 cases) as well as participants who reported incomprehensible demographic answers (16 cases). Accordingly, our final sample consisted of 274 players. In the combined sample the majority of participants were male $(\mathrm{n}=185)$. The mean age was roughly 29 years $(M=29.26, S D=6.95)$, and most participants' held a bachelor's degree (62\%). In addition, participants were either North American ( $\mathrm{n}=$ $155)$ or Indian $(n=119)$, reported playing video games for approximately just under 8 hours per week $(M=$ $7.95, S D=7.51$ ), their playing experience was a little more than 3 years as an average $(M=3.19, S D=$ 2.00 ), and 117 participants primarily played League of Legends and 157 Dota 2. The Table 2 describes the demographic information in relation to the two cultures (North America vs. India).

\section{Results}

\subsection{Influence of the cultural dimensions}

Referring to RQ1 (How does the frequency of toxicity differ between different cultural settings?), we conducted an independent samples t-test to compare TB in NA (i.e., low in collectivism and power distance and high in indulgence) and India (i.e., high in collectivism and power distance and low in indulgence). The requirements for using the test were met, as Levene's test suggested equality of variance $(F=$ $1.62, p=.20)$ and both skewness $(-.13)$ and kurtosis (-1.25) of TB indicated no violations of the required condition of normal distribution. Results showed a significant difference between the two groups $(F(1,272)=8.03, p=.001)$, with scores for India ( $M=4.75, S D=1.54)$ showing significantly higher levels of self-reported TB than the NA group ( $M=$ $3.20, S D=1.62$ ). These results suggest that the level of collectivism, power distance, and indulgence have an influence on the occurrence of TB in both cultures in our sample. The differences in TB between the two samples are quantified in Figure 2.

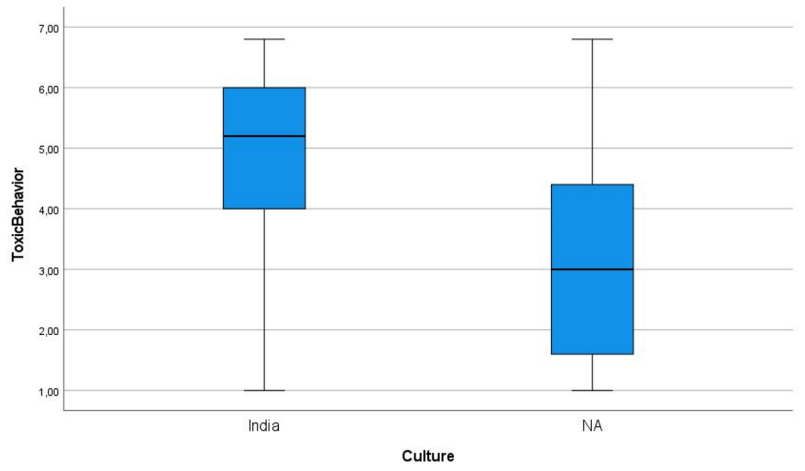

Figure 2. A box plot depicting self-reported TB among the two samples 


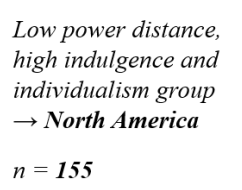

$* * * p \leq 0.01$
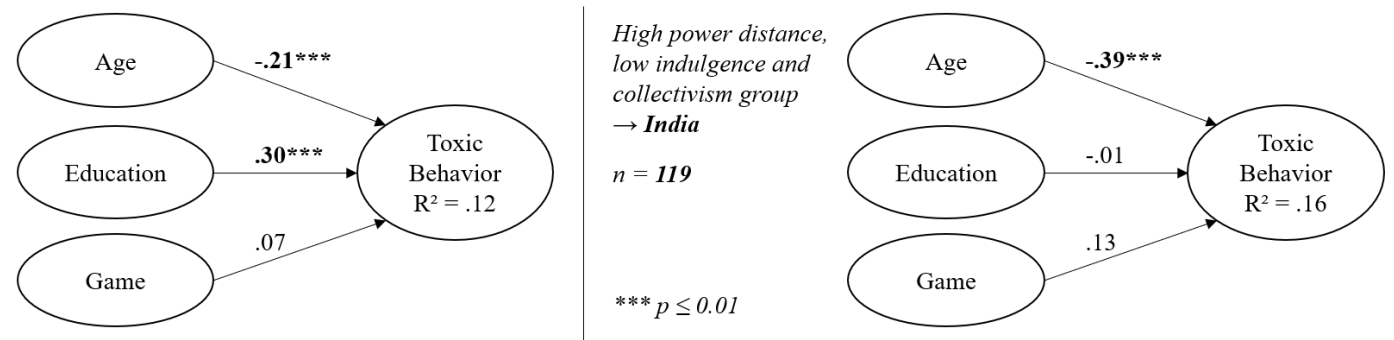

Figure 3. Comparison of potential factors of TB between North American and Indian MOBA players $(n=274)$

\subsection{Influence of demographic variables}

To obtain relevant findings regarding RQ2 (What influence do demographic variables have on the occurrence of toxicity in different cultural settings?), we proceeded in three subsequent steps.

First, we conducted a multiple regression analysis with demographic (e.g., age, gender, education) and control variables (e.g., playtime, playing experience, game type) as predictors to determine which variables exerted influences on the dependent variable toxic behavior. The Durbin-Watson test statistic $(d=1.78)$ indicated no violations of autocollinearity [44] and the regression equation showed a significant result $(F(6,267)=12.23, p=.001)$ and explained $20 \%$ of the variance of toxic behavior. In particular, age $(\beta=-.31, p=.001)$, education $(\beta=.27, p=.001)$, and game type $(\beta=.16, p=.01)$ had significant effects. For the variables gender, playtime, and playing experience, the analyses revealed no relevant relationships $(\beta \leq .13, p \geq .08)$. Accordingly, we excluded them from the subsequent steps of our analysis.

In the second step, we wanted to explore the significant predictors of TB identified in the first step: (1) age; (2) education; and (3) game type; in relation to the two cultural groups.

In the case of age, we performed a independent-samples t-test to test the cultural differences. Levene's test $(F \geq 21.26, p \leq .001)$ showed that there was a violation of the required conditions. Accordingly, we used the test statistic of unequal variances instead. Results showed a significant difference $(F(1,240)=-5.32, p=.001)$, with significantly lower scores for India $(M=27.00, S D=$ 4.11) than NA $(M=31.00, S D=8.10)$.

In case of education, we carried out another independent-samples $\mathrm{t}$ test. Levene's test $(F \geq$ $66.17, p \leq .001$ ) showed again a violation of the required conditions. Thus, similarly as before, we used the test statistic of unequal variances instead. Results showed a significant difference $(F(1,232)=4.94, p=$ $.001)$ with higher scores for India $(M=7.11, S D=$ .697) compared to NA ( $M=6.46, S D=1.44)$.

To test the relationship between game type (i.e., LoL or Dota 2) and the two cultural groups of NA and India, a chi-square test of independence was performed. The relation between both variables was significant, X2 (1, $\mathrm{N}=274)=11.59, \mathrm{p}=.001$, whereby players in NA reported to play League of Legends more frequently than expected (52\% vs. $43 \%)$ and players from India to play Dota 2 more frequently than expected (71\% vs. $59 \%)$.

Third, we wanted to find out more about the direction of the influence of age, education, and game type in relation to the cultures NA and India. Accordingly, we divided the data set into a low collectivism and power distance and high indulgence group (i.e., NA) and a high collectivism and power distance and low indulgence group (i.e., India) and conducted two regression analyses using age, education, and game type as predictors to explain the dependent variable toxic behavior for both groups. The Durbin-Watson test statistic indicated no violations regarding autocollinearity for either group $(\mathrm{d}=1.94, \mathrm{~d}=$ 2.11). The first regression equation for the NA group showed a significant result $(F(3,115)=8.06, p<$ 0.001 ) and explained $12 \%$ of the variance in toxic behavior. Among these, age $(\beta=-.21, p=.007)$ and education $(\beta=.30, p=.001)$ had significant effects (opposed to game type $(\beta=.07, p=.37)$ ). The second regression for the group from India showed a significant result as well $(F(3,115)=8.40, p<.001)$ and explained $16 \%$ of the variance in toxic behavior, with the variable age $(\beta=-.39, p=.001)$ showing a significant effect (in contrast to education $(\beta=-.01, p=.93)$ and game type $(\beta=.13, p=.14))$.

Accordingly, we found empirical evidence that age reduced the occurrence of TB in both samples (NA and India). But surprisingly, education increased TB in the North American sample. These findings are summarized in Figure 3. 


\section{Discussion}

\subsection{Key Findings}

Based on our results, we summarize our key findings with the following three points:

First, there are quantifiable differences in self-reported TB between national cultures. These findings may be explained by the the theoretical perspective of MNC [13, 12]. From this viewpoint, it can be argued that residents in countries such as India that is lean more to fulfill collective social roles and expectations to a greater extent in their daily lives - compared to NA. The online space provides the opportunity to cope with corresponding conditions in an anonymous matter, which links this finding to the online disinhibition effect [25]. Additionally, this finding is in line with the insights from Kwak et al. [17] who showed that TB differs significantly by server region on the more specific level of national cultures.

Second, there is a difference in preference for game type between national cultures, which is something we can observe quantitatively in the real world. Games like Starcraft: Brood War became popular in South Korea [45], but nowhere else to the same extent. In our sample, respondents from NA preferred to play LoL and players from India preferred Dota 2. From MNC [13]'s perspective, this can be attributed to differences in the cultural dimension of indulgence, which differs significantly between NA and India (see Figure 1). Accordingly, LoL values accessibility and fun, which should be associated with higher levels of indulgence (i.e., NA), whereas Dota 2 values depth at the expense of convenience.

Third, there are different explanations for the occurrence of toxicity for both cultures. Age, as suspected in previous literature on psychosocial development [46] and also on TB [11], had a negative influence on toxicity in both cultures, which can be attributed to the adoption of social norms and a socially acceptable self-presentation with increasing age. In contrast, education had a positive effect on TB only in the NA group. This finding is peculiar and we cannot offer an exhaustive explanation based on our empirical work or theoretical perspective. A potential starting point could be to build on existing findings that showed a negative relationship between education (specifically the academic self-concept of players) and the motivation to play and the subsequent use of MOGs [47]. Accordingly, playing MOGs might be more important for players with a lower significance of academic competencies, which increases the relevance of good performance during gameplay and subsequently makes the expression of toxicity more likely. Furthermore, in the case of India, the majority of participants $(n=93)$ had a BA as their highest academic degree, resulting in a significant variance restriction that prevents adequate findings regarding our statistical analysis.

\subsection{Theoretical implications}

This work offers theoretical contributions to the MNC [13], TB in video games [4, 5], and human online behavior in general. We elaborate on these contributions in the following three paragraphs.

First, we have extended the external validity of the national culture model by showing that countries that differ in terms of their cultures (particularly individualism/collectivism, power distance, and indulgence) require nuanced explanations for the occurrence of TB that add value to the current picture of empirical knowledge. Nonetheless, we encourage future research to explicitly measure the cultural dimensions. In addition, we recommend including all six dimensions of national identities for a chance to derive more explicit and holistic findings (and perhaps more surprising results) regarding MNC [13].

Second, our work contributes to the literature on TB in video games $[4,17]$ and also more broadly to work on online disinhibition [25]. Previous literature on TB in MOGs has focused predominantly on the influence of situational conditions $[6,8,9,11,16]$, attitudes [5], experiences [5], or motivations [10, 11] on $T B$, but neglected dispositional determinants such as culture and demographics as antecedent variables. Our work shows that these variables are indeed very important in explaining variances in TB. Collectivism, power distance, and forbearance explained a substantial amount of the variance in TB, which is particularly relevant given that global MOGs are similar across cultures to date, with little localization besides translation. With these findings, we extend previous work on TB and suggest that holistic models for understanding TB should consider culture-related variables more strongly. Our work also carries the important theoretical suggestion that the culture of the sample in game studies needs to be acknowledged when drawing conclusions from the results of empirical work.

Finally, Suler argues that there are several potential reasons as to why people behave more poorly in online compared to offline contexts [25]. These include (1) anonymity; and (2) invisibility; leading to lack of responsibility, (3) asynchronicity; (4) issues arising from not seeing others' facial expressions and body language; (5) lack of authority; and (6) hindered ability to imagine others' reactions [25]. Our findings can be 
understood as indicators that the relationships between constructs in the original study might be confounded with variables such as culture that have not been holistically captured. Accordingly, we recommend to consider interaction and mediation relationships more strongly. However, further work on this topic is warranted before conclusive associations can be drawn.

\subsection{Implications for game design}

From our findings, we also derive some implications at the level of multiplayer online game design. In doing so, we extend the knowledge of user-centric metrics - either at the individual or target group level - to capture TB by showing that game designers designing countermeasures against TB need to focus not only on in-game and online culture, but also consider the offline culture of players. Using culture as a determinant seems particularly promising because this data is already available and can be collected with little effort. Additionally, the insights of our study add value regarding the diverging discussion on determinants of effective gamification and serious game design [48]. On a more general level, we argue that game designers should expand the scope of available data on players' personalities and social environments to develop user-centered solutions. Specifically, we derive two important insights from our empirical work that game designers might find useful in designing countermeasures for TB.

First, the level of TB between players is influenced by national culture. It is important to note that national culture is not malleable, and therefore interventions should work through understanding the culture, not through trying to change it. In other words, designers should look at region-specific solutions and strategies to mitigate $\mathrm{TB}$ in global MOGs. For example, in countries with higher collectivism, social norming mechanisms such as introducing in-game nudges toward collaborative behavior and displaying honor levels on player profiles to other players could mitigate the effect of online disinhibition. In contrast, currently employed measures such as reporting features $[16,22]$ or penalties $[17,22]$ preserve anonymity and may fail to achieve the desired effect in collectivist regions.

Second, young age is associated with TB. Designers might consider whether curbing $\mathrm{TB}$ can be combined with educational opportunities and encouragement of personal growth in their players. For example, in-game social praise for collaborative behavior could serve to educate younger players to deal with tilting in a non-offensive manner. Meta-analyses based on cognitive evaluation theory show that informational reinforcements, such as verbal praise or status symbols, are significantly more effective in influencing behavior than rewards and punishments [49] currently used by game designers $[17,22]$.

\subsection{Limitations and future work}

The model and hypothesis tested in this study might have missed some important background factors, such as ethnicity, duration of gaming sessions, average spending in games, religion, and income level, as they were not examined. Participants were recruited through Amazon's MTurk, meaning they were self-selected, and although participants were specifically asked for those who had experience with MOBA games, there may have been some responses that participated only because of the small monetary reward provided by the platform and were not captured in the initial screening of responses, which discarded responses that did not pass the control questions. Participants also represented only adult gamers, and since younger age was found to be positively correlated with $\mathrm{TB}$, it may be fruitful if future similar studies could include underage gamers.

Some limitations are also associated with the games studied. LoL and Dota 2 are known for TB directed against their own team members. However, such TB does not occur in all games. For example, recent work with players of Pokémon GO and Ingress showed that members of certain teams developed prejudices and negative feelings toward opposing team members with whom they were in conflict [50], but not toward members of the same team. This difference suggests that constantly changing teams (as in LoL and Dota 2) increase TB towards teammates, while static teams (as in Pokémon GO) decrease TB as teammates get to know and trust each other. The idea that constantly fluctuating teams induce TB is an interesting avenue for future work.

Finally, in the NA sample, a high level of education was positively associated with self-reported TB. Such surprising results should be replicated in the future and, if confirmed, studied in further detail. One avenue for future research could be to draw on negative behaviors similar to TB in other contexts (e.g., riots and protests) and see if national culture, as described by the MNC [13], yields similar results in these settings.

\section{Conclusions}

In this study, we investigated the role of two different cultures (NA and India) consulting the model of national culture (consisting of the six cultural dimensions power distance, individualism/collectivism, masculinity, uncertainty avoidance, long term orientation, and 
indulgence) and demographic variables in explaining toxicity in two popular MOGs: LoL and Dota 2. We discovered that there was a statistically significant difference in the occurrence of TB between two cultures (higher levels of TB in India). One of the given explanations for this could be that countries such as India that lean more to fulfill collective social roles and expectations to a greater extent in their daily lives - compared to NA, which leads to differences in responsibility regarding actions in an anonymous environment. Additionally, both cultures showed differences in terms of their game preferences (players from NA preferred LoL and players from India Dota 2). We attributed this finding to differences in the cultural dimension of indulgence, which differs significantly between NA (higher level of indulgence) and India (lower levels of indulgence) and the different priorities of both games (LoL uses accessibility and fun and Dota 2 depth at the expense of convenience). In our sample of NA players $(n=155)$, education had a positive effect on toxicity. This finding was surprising and further work on this is warranted, including replication of the finding. Finally, we confirmed that age reduced TB in both samples (both national cultures). This universal finding is consistent with research on psychosocial development and how aggression decreases with age. Therefore, games with a young player base are more likely to experience issues with TB.

\section{References}

[1] Newzoo, "Video game market value worldwide from 2012 to 2023," [ONLINE], available at: https://www.statista.com/statistics/292056/video-gamemarket-value-worldwide/], 2020.

[2] S. Laato, S. Rauti, A. N. Islam, and E. Sutinen, "Why playing augmented reality games feels meaningful to players? the roles of imagination and social experience," Computers in Human Behavior, p. 106816, 2021.

[3] H. Park and K.-J. Kim, "Social network analysis of high-level players in multiplayer online battle arena game," in International Conference on Social Informatics, pp. 223-226, Springer, 2014.

[4] B. Kordyaka, M. Klesel, and K. Jahn, "Perpetrators in league of legends: Scale development and validation of toxic behavior," in Proceedings of the 52nd Hawaii International Conference on System Sciences, 2019.

[5] B. Kordyaka, K. Jahn, and B. Niehaves, "Towards a unified theory of toxic behavior in video games," Internet Research, 2020.

[6] K. Shores, Y. He, K. Swanenburg, R. Kraut, and J. Riedl, "The identification of deviance and its impact on retention in a multiplayer game," in Proceedings of the 17th ACM conference on Computer supported cooperative work \& social computing, (New York, NY, USA), pp. 1356-1365, ACM, 2014.

[7] J. Lin, "The science behind shaping behavior in online games," in [ONLINE] available at:
https://www.gdcvault.com/play/1017940/The-ScienceBehind-Shaping-Player, accessed 12th of April, 2021, gdcvault, 2021.

[8] C. Shen, Q. Sun, T. Kim, G. Wolff, R. Ratan, and D. Williams, "Viral vitriol: Predictors and contagion of online toxicity in World of Tanks," Computers in Human Behavior, vol. 108, p. 106343, 2020.

[9] S. Türkay, J. Formosa, S. Adinolf, R. Cuthbert, and R. Altizer, "See No Evil, Hear No Evil, Speak No Evil: How Collegiate Players Define, Experience and Cope with Toxicity," in Proceedings of the 2020 CHI Conference on Human Factors in Computing Systems, (New York, NY, USA), pp. 1-13, ACM, 2020.

[10] L. Achterbosch, C. Miller, and P. Vamplew, "A taxonomy of griefer type by motivation in massively multiplayer online role-playing games," Behaviour and Information Technology, vol. 36, no. 8, pp. 846-860, 2017.

[11] M. Lemercier-Dugarin, L. Romo, C. Tijus, and O. Zerhouni, "'who Are the Cyka Blyat?" How Empathy, Impulsivity, and Motivations to Play Predict Aggressive Behaviors in Multiplayer Online Games," Cyberpsychology, Behavior, and Social Networking, vol. 24, no. 1, pp. 63-69, 2021.

[12] G. Hofstede, "Dimensionalizing Cultures: The Hofstede Model in Context," Online Readings in Psychology and Culture, vol. 2, no. 1, pp. 116-130, 2011.

[13] G. Hofstede, "Riding the waves of commerce: A test of trompenaars' "model" of national culture differences," International journal of intercultural relations, vol. 20, no. 2, pp. 189-198, 1996.

[14] J. de Mesquita Neto and K. Becker, "Relating conversational topics and toxic behavior effects in a MOBA game," Entertainment Computing, vol. 26, pp. 10-29, 2018.

[15] J. Neto, K. Yokoyama, and K. Becker, "Studying toxic behavior influence and player chat in an online video game," in Proceedings of the International Conference on Web Intelligence, pp. 26-33, ACM, 2017.

[16] Y. Kou, "Toxic Behaviors in Team-Based Competitive Gaming: The Case of League of Legends," in Proceedings of the Annual Symposium on Computer-Human Interaction in Play, (New York, NY, USA), pp. 81-92, ACM, 2020.

[17] H. Kwak, J. Blackburn, and S. Han, "Exploring Cyberbullying and Other Toxic Behavior in Team Competition Online Games," in Proceedings of the 33rd Annual ACM Conference on Human Factors in Computing Systems, (New York, NY, USA), pp. 3739-3748, ACM, 2015.

[18] S. Einarsen, H. Hoel, and C. Cooper, Bullying and Emotional Abuse in the Workplace. CRC Press, 2002.

[19] P. Smith, J. Mahdavi, M. Carvalho, S. Fisher, S. Russell, and N. Tippett, "Cyberbullying: Its nature and impact in secondary school pupils," Journal of Child Psychology and Psychiatry and Allied Disciplines, vol. 49, no. 4, pp. 376-385, 2008.

[20] C. Monge and T. O'Brien, "Effects of individual toxic behavior on team performance in League of Legends," Media Psychology, vol. ahead-of-p, jan 2021.

[21] D. Ewoldsen, C. Eno, B. Okdie, J. Velez, R. Guadagno, and J. Decoster, "Effect of playing violent video games cooperatively or competitively on subsequent cooperative behavior," Cyberpsychology, Behavior, and Social Networking, vol. 15, no. 5, pp. 277-280, 2012. 
[22] S. Adinolf and S. Turkay, "Toxic Behaviors in Esports Games," in Proceedings of the 2018 Annual Symposium on Computer-Human Interaction in Play Companion Extended Abstracts, (New York, NY, USA), pp. 365-372, ACM, 2018.

[23] K. Emmerich, A. Krekhov, and J. Krüger, “"Pls Uninstall": On the Interplay of the COVID-19 Pandemic and Toxic Player Behavior in Competitive Gaming," in Extended Abstracts of the 2020 Annual Symposium on Computer-Human Interaction in Play, (New York, NY, USA), pp. 224-228, ACM, 2020.

[24] B. Maher, "Can a video game company tame toxic behaviour?," Nature, vol. 531, pp. 568-571, mar 2016.

[25] J. Suler, "The online disinhibition effect," Cyberpsychology \& behavior, vol. 7, no. 3, pp. 321-326, 2004.

[26] D. Nieborg and T. Poell, "The platformization of cultural production: Theorizing the contingent cultural commodity," New Media \& Society, vol. 20, pp. 4275-4292, Nov. 2018

[27] M. Lehtonen, A. Ainamo, and J. Harviainen, "The four faces of creative industries: visualising the game industry ecosystem in Helsinki and Tokyo," Industry and Innovation, vol. 27, pp. 1062-1087, Oct. 2020.

[28] H. Jenkins, Convergence Culture: Where Old and New Media Collide. NYU Press, 2006.

[29] M. Meriläinen, R. Aurava, A. Kultima, and J. Stenros, "Game Jams for Learning and Teaching: A Review," International Journal of Game-Based Learning (IJGBL), vol. 10, no. 2, pp. 54-71, 2020. ISBN: 9781799807483.

[30] W. Hamilton, O. Garretson, and A. Kerne, "Streaming on Twitch: Fostering Participatory Communities of Play within Live Mixed Media," in Proceedings of the SIGCHI Conference on Human Factors in Computing Systems, CHI '14, (New York, NY, USA), pp. 1315-1324, Association for Computing Machinery, 2014. event-place: Toronto, Ontario, Canada.

[31] L. Hjorth and D. Chan, Gaming Cultures and Place in Asia-Pacific. Routledge, 2009.

[32] M. Cwil and W. T. Howe, "Cross-Cultural Analysis of Gamer Identity: A Comparison of the United States and Poland," Simulation \& Gaming, vol. 51, pp. 785-801, Aug. 2020.

[33] F. Mäyrä, An Introduction to Game Studies. SAGE Publications, 2008.

[34] C. A. Steinkuehler, "Why Game (Culture) Studies Now?," Games and Culture, vol. 1, pp. 97-102, Jan 2006.

[35] A. Shaw, "What Is Video Game Culture? Cultural Studies and Game Studies," Games and Culture, vol. 5, pp. 403-424, Oct. 2010. Publisher: SAGE Publications.

[36] M. Wolf, ed., Video Games Around the World. MIT Press, 2015.

[37] T.-J. Yoon and H. Cheon, "Game playing as transnational cultural practice: A case study of Chinese gamers and Korean MMORPGs," International Journal of Cultural Studies, vol. 17, pp. 469-483, Oct. 2013. Publisher: SAGE Publications Ltd.

[38] A. Elmezeny and J. Wimmer, "Games without Frontiers: A Framework for Analyzing Digital Game Cultures Comparatively," Media and Communication, vol. 6 , pp. 80-89, June 2018.
[39] V. Šisler, J. Švelch, and J. Šlerka, "Video Games and the Asymmetry of Global Cultural Flows: The Game Industry and Game Culture in Iran and the Czech Republic," International Journal of Communication, vol. 11 , no. 0 , p. $23,2017$.

[40] N. Taylor, "Play Globally, Act Locally: The Standardization of Pro Halo 3 Gaming," International Journal of Gender, Science and Technology, vol. 3, Mar. 2011.

[41] NEWZOO, "2020 Global Games Market Report," tech. rep., NEWZOO, 2020.

[42] T. Wijman, "The India Opportunity: Tapping into One-Tenth of the World's Gamers," consumer insights, NEWZOO, 2018

[43] C. DiStefano, M. Zhu, and D. Mîndrilă, "Understanding and using factor scores: Considerations for the applied researcher," Practical Assessment, Research and Evaluation, vol. 14, no. 20, 2009.

[44] J. Cohen, P. Cohen, S. G. West, and L. S. Aiken, Applied multiple regression/correlation analysis for the behavioral sciences. Routledge, 2013.

[45] J.-S. Huhh, "Culture and business of pc bangs in korea," Games and Culture, vol. 3, no. 1, pp. 26-37, 2008.

[46] D. Rosenthal, R. Gurney, and S. Moore, "From trust on intimacy: A new inventory for examining erikson's stages of psychosocial development," Journal of Youth and Adolescence, vol. 10, no. 6, pp. 525-537, 1981.

[47] B. Kordyaka, K. Jahn, M. Müller, and B. Niehaves, "The comparative self: Understanding the motivation to play and the subsequent video game use," in Proceedings of the DiGRA Conference, 2019.

[48] J. Krath, L. Schürmann, and H. F. von Korflesch, "Revealing the theoretical basis of gamification: A systematic review and analysis of theory in research on gamification, serious games and game-based learning," Computers in Human Behavior, p. 106963, 2021.

[49] R. Ryan and E. Deci, Self-Determination Theory. Basic Psychological Needs in Motivation, Development and Wellness. New York, London: The Guilford Press, 2017.

[50] S. Laato, N. Inaba, M. Paloheimo, and T. Laajala, "Group polarisation among location-based game players: an analysis of use and attitudes towards game slang," Internet Research, 2021. 